\title{
Modelo de simulação baseada em agentes para avaliar política de distribuição de cargas urbanas do e-commerce
}

\author{
Roberta Alves ${ }^{1}$, Renato da Silva Lima², Alexandre Ferreira de Pinho ${ }^{3}$, Jose Holguín-Veras ${ }^{4}$ \\ 1Universidade Federal de São João del Rei, MG - Brasil, robertalvess@hotmail.com \\ 2Universidade Federal de Itajubá, MG - Brasil, rslima74@gmail.com \\ 3Universidade Federal de Itajubá, MG - Brasil, pinho@unifei.edu.br \\ ${ }^{3}$ Rensselaer Polytechnic Institute - Center for Infrastructure, Transportation, and the Environment, EUA, jhv@rpi.edu
}

\section{Recebido:}

28 de abril de 2019

Aceito para publicação:

8 de outubro de 2019

Publicado:

12 de novembro de 2019

Editor de área:

Claudio Barbieri da Cunha

\section{Palavras-chaves:}

Logística Urbana;

Comércio Eletrônico;

Simulação Baseada em Agentes;

City Logistics.

\section{Keywords:}

Urban Logistics,

E-commerce,

Agent Based Simulation,

City logistics.

DOI:10.14295/transportes.v27i3.2034

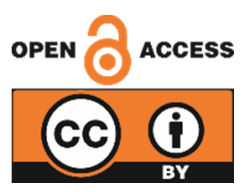

\begin{abstract}
RESUMO
Este estudo tem como objetivo avaliar a implantação das Estações de Entrega Automática (EEA) como solução para a entrega da última milha, considerando o comportamento e a interação entre os stakeholders do e-commerce. No Brasil, a maior parte maior das entregas são à domicílio e assistidas. Isso resulta em um grande número de entregas com falha e tentativas de reentrega. As Estações de Entrega Automática (EEA) representam uma solução para reduzir a quantidade de devoluções e consolidar a entrega de mercadorias. Simulamos quatro cenários em Belo Horizonte, Brasil, variando a implementação da EEA e a exclusão das tentativas de devolução. Os EEAs trazem melhorias em termos de redução de reentregas e das distâncias percorridas pelos caminhões em até $46 \%$.
\end{abstract}

\begin{abstract}
This study aims to explore how Agent-Based Simulation Models (ABSM) can help reduce the impact of e-commerce deliveries in urban logistics. In Brazil, e-commerce deliveries are all person-assisted. This results in a large number of failed deliveries and redelivery attempts. Automatic Delivery Stations (EEA) represent a solution to reduce the amount of redeliveries and consolidate the delivery of goods. We simulated four scenarios in Belo Horizonte, Brazil, by varying the EEA implementation and the exclusion of the redelivery attempts. The EEAs bring improvements in terms of reducing redeliveries and the distances that the trucks traveled by up to $46 \%$.
\end{abstract}

\section{INTRODUÇÃO}

A tendência de crescimento do comércio eletrônico é mundial, segundo o portal eMARKETER (2019), o e-commerce apresentou uma média de crescimento de $24 \%$ no mundo todo em 2018 , atingindo uma marca de 2,9 trilhões de dólares em vendas. As vendas online apresentam crescimento maior que o varejo tradicional em praticamente todos os países que já operam o comércio eletrônico. Na América Latina o crescimento foi de 17,9\% com relação ao mesmo período de 2017. 0 Brasil é o mercado mais desenvolvido em termos de e-commerce na América Latina. 0 e-commerce brasileiro cresceu $12 \%$ e faturou $\mathrm{R} \$ 53,2$ bilhões em 2018 em comparação com o ano de 2017 (Ebit, 2019). 
Quanto maior o crescimento das compras on-line, maior é a demanda por entregas individuais, implicando em um número maior de veículos de carga circulando em áreas urbanas (Cardenas et al., 2017). Sendo assim, maiores são os desafios enfrentados na distribuição de mercadorias (Morganti, et al., 2014; Visser et al., 2014), com efeito direto nos sistemas logísticos em áreas urbanas, onde o congestionamento e a acessibilidade são fatores cruciais (Alves et al., 2019; Holguín-Veras et al., 2016; Kedia et al., 2017).

No caso do mercado $\mathrm{B} 2 \mathrm{C}^{\mathrm{i}}$ (bussiness-to-costumer) as entregas assistidas à domicílio (EAD) são geralmente preferidas pelo consumidor. Este tipo de entrega constitui a solução mais problemática em termos de custos de serviços e programação (Morganti, et al., 2014). Nesta modalidade, os entregadores realizam várias paradas ao longo de uma viagem e dependem da presença de uma pessoa no local para realizar a entrega (Oliveira, et al., 2017). A ausência dessa pessoa resulta em falha na entrega. De acordo com Song et al. (2009) 25\% das entregas não são realizadas na primeira tentativa.

Assim como na maioria das cidades latino-americanas, no Brasil as entregas do comércio eletrônico são em sua maioria assistidas, ou seja, necessitam de uma pessoa no endereço indicado (geralmente casa ou trabalho) para receber as mercadorias (Ebit, 2019; Maere, 2017). Em grande parte dos países europeus e nos EUA, quando não há alguém no endereço para receber as mercadorias, estas são deixadas sem supervisão na calçada (on-street) (Morganti, Seidel, et al., 2014). Segundo Oliveira et al. (2017), entregas sem supervisão não são ainda viáveis no Brasil, pois representam "uma tentação para ladrões". Esta situação, aliada a dificuldade de estipular janelas de entregas para os clientes, contribui para aumentar as entregas falhas (Visser et al., 2014).

As entregas a domicílio apresentam desvantagens para o consumidor que necessita de flexibilidade e para o entregador que busca otimizar a sua distribuição de mercadorias. Iniciativas de city logistics que ofereçam uma alternativa à esse tipo de entrega e minimizem os inconvenientes causados por ela têm sido objeto de estudo de várias pesquisas (Iwan et al., 2016; Liu et al., 2017). Estes autores sugerem como uma solução para este problema a consolidação das entregas da última milha. Neste contexto, encontram-se as Estações de Entrega Automática (EEA), que já foram implementados em vários países(Iwan et al., 2016; Morganti, Dablanc, et al., 2014; Weltevreden, 2008). As estações são armários automatizados que os consumidores podem utilizar para retirar e/ou retornar suas compras realizadas online.

Contudo, Taniguchi et al. (2012) afirmam que antes de implementar qualquer iniciativa de city logistics, é preciso entender o comportamento (ações e objetivos) dos stakeholders envolvidos na logística urbana e a interação entre eles. A Modelagem e Simulação Baseada em Agentes (MSBA) permite captar a dinâmica do sistema em situações onde outros tipos de simulação não conseguem lidar de forma adequada com os agentes e variáveis associadas a eles (Oliveira et al., 2016). Isso se deve ao fato de a MSBA utilizar em sua construção os objetivos e comportamentos individuais dos agentes (Elbert e Friedrich, 2018; Firdausiyah et al., 2019). Alguns estudos tem obtido tal feito ao utilizar este tipo de simulação em logística urbana (Alho et al., 2017; Oliveira, et al., 2017; Roorda et al., 2010; Teo et al., 2012). Nestes trabalhos, os stakeholders envolvidos na logística urbana são modelados como agentes capazes de tomar decisões e interagir entre si e com o meio, ao se deparar com uma situação pré-determinada (Davidsson et al., 2005). No entanto, a maior parte dos trabalhos de MSBA lida com políticas que buscam de alguma forma restringir a circulação de veículos, sem analisar os impactos da aplicação de EEA no ambiente urbano. 
Para tanto, este estudo tem como objetivo avaliar a implantação das EEAs como solução da última milha, considerando o comportamento e interação entre os stakeholders do e-commerce. As simulações foram realizadas para a região central de Belo Horizonte, MG, Brasil, a partir de cenários que procuram alternar as medidas para reduzir as falhas nas entregas. A MSBA construída foi fundamentada nos objetivos e comportamentos individuais dos stakeholders do transporte urbano, modelados como agentes.

\section{REFERENCIAL TEÓRICO}

\subsection{E-commerce e transporte urbano de mercadorias}

As estações de entrega automática (EEAs) são um tipo de ponto de coleta e entrega do inglês Collection-and-Delivery Points (CDPs) e funcionam sem supervisão. As EEAs são armários automatizados operados a partir de um código eletrônico, onde os entregadores utilizam este código para informar os clientes sobre a situação de suas encomendas (Iwan et al., 2016).

A maior parte das entregas do comércio eletrônico requerem uma pessoa para receber a encomenda. No entanto, pode vir a acontecer de os consumidores não se encontrarem em suas residências, quando um pacote é posto na rota de entrega aos clientes, e ocorrer então uma "falha" na entrega (Maere, 2017). Nesse caso, estabelece-se a necessidade de nova(s) viagem (s) até a residência do cliente. Se mesmo depois de uma terceira tentativa a entrega não puder ser concluída, os operadores logísticos podem oferecer diferentes opções para os clientes. Uma alternativa é deixar a encomenda com um vizinho, deixar fora das instalações (não seguro) ou, entregar o item em um local próximo, como por exemplo em uma EEA. Quando nenhuma das opções está disponível, o item é retornado ao remetente. Isso implica em altos custos que devem ser arcados, ou pelo operador logístico, ou, pelo varejista online ou consumidor (Morganti, Seidel, et al., 2014).

De acordo com Weltevreden (2008), com o uso das EEAs, os entregadores podem alcançar economia de tempo e combustível, tanto para primeiras entregas quanto para entregas falhas, pois não seria necessário retornar ao endereço do consumidor para uma segunda ou terceira tentativa de entrega. Neste cenário, os EEA se mostram como uma solução atraente para o contexto brasileiro, pois apresentam segurança e reduzem as falhas das entregas (de Oliveira, Morganti, et al., 2017).

As estações de entrega automáticas são o foco desse trabalho, especialmente por possuírem uma janela de tempo de funcionamento ampla. Apresentam outras vantagens: (i) o cliente não precisa ficar o dia todo em casa esperando a entrega da mercadoria (ii) alta taxa de satisfação dos clientes; (iii) apresentam alto nível de segurança no serviço; (iv) reduz distâncias extras percorrida em função de reentregas; (v) possibilitam a otimização das rotas; (vi) possibilitam o rastreamento da entrega; (vii) possibilitam uma melhor utilização dos veículos (viii) aumentam a atratividade dos locais onde estão instalados e (ix) o maior benefício: 100\% das entregas são realizadas (McLeod et al., 2006; Morganti, Dablanc, et al., 2014; Weltevreden, 2008).

Apesar das inúmeras vantagens, quando mal dimensionado esse sistema pode gerar viagens extras de clientes em seus veículos privados para buscar seus pedidos. Para minimizar este problema, Dell'Amico e Hadjidimitriou (2012) reforçam que a localização adequada é importante para o sucesso do sistema, pois pode facilitar o encadeamento de viagens: o cliente pode conciliar uma ida a EEA com seu trajeto diário (Liu et al., 2017; Weltevreden, 2008).

Grande parte dos estudos que investigam esta alternativa para a última milha se preocupam em: (1) conceituar o tema (Cullinane, 2009; Visser et al., 2014); (2) caracterizar a demanda 
destes serviços (Iwan et al., 2016; Kedia et al., 2017; de Oliveira, Morganti, et al., 2017; Weltevreden, 2008) e (3) desenvolver métodos e modelos matemáticos para analisar a potencial economia da utilização dos pontos de coleta (Arnold et al., 2018; I. D. Cardenas et al., 2017; Liu et al., 2017).

Arnold et al. (2018) estudaram as viagens resultantes das compras online no contexto da cidade de Antwerp. Eles simularam o cenário atual, que trata de entregas à domicílio realizadas por furgões, e mais três cenários, que incluem o uso de CDPs, o uso de bicicletas de carga e um sistema híbrido. 0 sistema híbrido foi identificado como o cenário que traria melhores resultados em termos de custos operacionais e externos. A simulação realizada por eles é uma simulação estática que é resolvida pelo problema de roteamento de veículos. A simulação estática, segundo Van Kolck (2010), não estima o resultado para os vários agentes envolvidos no transporte urbano de mercadorias, pois adapta o interesse individual de cada agente para perspectivas e conceitos globais da sociedade.

Os estudos descritos anteriormente mostram a importância das EEA para melhorar a eficiência da distribuição de bens urbanos. Contudo, não evidenciam as implicações que esta iniciativa pode trazer para todos os envolvidos no frete urbano. Este estudo busca investigar a implantação destas estações automáticas em Belo Horizonte considerando as políticas locais por meio de modelagem e simulação baseada em agentes.

\subsection{Simulação baseada em agentes}

De acordo com Taniguchi et al. (2012) a maioria dos modelos que simulam a logística urbana não consideram os interesses individuais de cada agente, adaptando os objetivos e perspectivas individuais para objetivos e perspectivas comuns. Dessa forma, os impactos das medidas da logística urbana não são estimados para os vários agentes envolvidos. Bons resultados têm sido alcançados ao se utilizar Modelos de Simulação Baseados em Agentes (MSBA) para avaliar o comportamento e reações dos atores urbanos ante implantação de iniciativas de city logistics. Esses modelos começaram a ser desenvolvidos nos últimos anos e geraram grande interesse na área de frete urbano (Davidsson et al., 2005; Taniguchi et al., 2012)

Os MSBA quando utilizados para representar o comportamento de vários agentes dentro de um sistema são comumente chamados de Sistemas Multi-agente (Multi-Agent System -MAS). De forma geral o MAS considera a interação de cada agente com o seu ambiente, seguindo os seus comportamentos e objetivos pré-estabelecidos. Um agente pode ser entendido como um sistema autônomo com um objetivo determinado, que opera assincronamente e, quando necessário, pode atuar de modo coordenado com outros agentes (Fox et al., 2000).

Em relação ao transporte urbano de mercadorias, existem vários interessados (varejista, transportadora, embarcador, cliente e etc) que possuem objetivos diferentes perante a movimentação de mercadorias. Por esta razão, os trabalhos nesse contexto que utilizam MSBA são tratados como Sistemas Multi-Agente (Davidsson et al., 2005) e em geral essa modelagem é utilizada para compreender e analisar a resposta dos diferentes agentes às medidas e inciativas de city logistics (Wangapisit et al., 2013).

Elbert e Friedrich, (2018) Firdausiyah, Taniguchi e Qureshi (2019) e van Heeswijk, Larsen e Larsen (2019) utilizaram a MSBA para avaliar as entregas em Centros de Consolidação Urbana (CCU) por diferentes perspectivas. Elbert e Friedrich (2018) consideraram cenários com políticas de restrição de circulação de veículos, van Heeswijk, Larsen e Larsen (2019) testaram 
várias combinações de medidas, Firdausiyah, Taniguchi e Qureshi (2019) focaram o estudo no comportamento do agente.

Marcucci et al. (2017) desenvolveram um modelo multi-agente para apoiar as decisões do transporte urbano de mercadorias em um ambiente de zona de baixa emissão de poluentes, em Roma. Schroeder et al. (2012) desenvolveram um modelo multi-agente e avaliaram cenários com diferentes políticas de entregas, onde as transportadoras e os prestadores de serviços logísticos são os agentes que, ao tomarem decisões conjuntas, conseguem melhorar ou não os seus processos logísticos. Os Autores apresentam os resultados para um exemplo teórico e representam o espaço urbano por meio de um GRID. Essa mesma abordagem de representação é adotada por Teo et al. (2012) para analisar a implantação de iniciativas e políticas públicas para o transporte urbano de cargas que controlem a poluição gerada pelos caminhões. Segundo os autores, o pedágio urbano trouxe melhores resultados ao fornecer uma maior redução da poluição em comparação com a precificação baseada na distância.

A capacidade do MSBA de capturar a hetoregeineidade dos vários stakeholders da logística urbana e suas interações é destacada por Sopha et al. (2016). Em seu trabalho, a simulação multi-agente é utilizada para resolver o problema de roteamento dinâmico de veículos. Oliveira et al. (2017a) relatam como o uso de MSBA pode auxiliar os atores de frete urbano a tomar decisões em situações de inundações em locais da rede, e com isso reduzir os impactos no transporte urbano de mercadorias.

A maior parte dos trabalhos que utiliza MAS como um sistema de decisão multi-agente e lidam com políticas que buscam de alguma forma restringir a circulação de veículos(Alho et al., 2017; Davidsson et al., 2005; Roorda et al., 2010) ou estudam algum caso especifico (Elbert e Friedrich, 2018; Firdausiyah et al., 2019; van Heeswijk et al., 2019). Nenhum desses trabalhos analisa os impactos da aplicação das EEAs no ambiente urbano por meio de MSBA, que é o foco desse trabalho. Para tanto, a MAS foi construída tendo como plataforma operacional o software Anylogic $®$ para analisar políticas de frete urbano para o comércio eletrônico.

\section{MODELAGEM E SIMULAÇÃO BASEADA EM AGENTES}

\subsection{Identificação da Situação-Problema}

Toda a modelagem foi realizada baseada em ações e interações dos stakeholders do processo, que são representados como agentes na simulação. 0 modelo foi desenvolvido para região conhecida como "Contorno" da cidade de Belo Horizonte, capital do estado de Minas Gerais. A região do contorno corresponde a região central de Belo Horizonte e recebe este nome pois é limitada pela avenida do contorno, esta região compreende os bairros: Barro Preto; Santo Agostinho; Lourdes; Savassi; Funcionários; Boa Viagem; e parte dos bairros Santa Efigênia e Floresta.

Fez-se uso de uma região concreta para poder atribuir uma demanda de clientes específica dessa região. Isso nos permitiu utilizar dados de estudos realizados anteriormente para caracterizar os agentes e suas preferências (Oliveira, et al., 2017). Além disso, as transportadoras que auxiliaram durante a construção e validação do modelo, com os dados referentes a modelagem do Agente Caminhão, atuam nesta região. Outro motivo para a escolha da região foi por se tratar de uma área economicamente relevante para a Belo Horizonte, apresenta uma alta urbanização, o que contribui para o surgimento de problemas relacionados a entregas de mercadorias nesta região.

Atualmente, as entregas do e-commerce em Belo Horizonte sua grande maioria domiciliares. No contexto brasileiro, essas entregas obedecem a política das três tentativas, ou seja, quando 
a entrega não acontece em uma primeira tentativa, podem acontecer até duas tentativas nos dias seguintes. Fica evidente que esta política gera mais viagens e consequentemente aumenta os custos do frete. Dessa forma, este estudo busca verificar se a implantação das EEAs pode contribuir para redução da política das três tentativas.

\subsection{Modelo conceitual}

Para a construção do modelo foram necessárias informações sobre o comportamento do agente que realiza uma ação, que geram então os outputs que permitem analisar os cenários. Estas informações foram obtidas de forma direta a partir de dados do Ebit (2018), da pesquisa realizada por Oliveira et al. 2017 e com transportadoras que atuam na região estudada, e a de forma indireta com base em dados obtidos da revisão de literatura. 0 modelo considera a interação dos quatro principais agentes envolvidos no processo de entregas de mercadorias do comércio eletrônico: (i) transportadora; (ii) loja do comércio eletrônico; (iii) EEAs e (iv) cliente. A modelagem de cada agente é apresentada a seguir.

\subsubsection{Modelagem dos Agentes}

\section{Modelagem do Cliente e Ponto de EEA}

A região do Contorno região possui cerca de 93.684 habitantes (IBGE, 2010). Como levantado por Oliveira et al. (2017), a porcentagem de clientes que realiza compras online é cerca de $30 \%$ da população, ou seja, 28.100 clientes. No modelo as residências dos 28.100 clientes foram localizadas dentro da região do contorno. Geograficamente estas residências foram dispostas de forma homogênea dentro da região.

O modelo é iniciado no momento em que os clientes realizam a compra, estes escolhem por receber seu pedido em casa ou nas EEAs, o software separa os clientes aleatoriamente respeitando o parâmetro de entrada adotado. Como nesta região não existem EEAs em funcionamento, estimamos a quantidade de clientes que estaria disposta a utilizar as EEAs baseado em Oliveira et al. (2017), onde os autores relatam que em Belo Horizonte $43 \%$ dos cliente do comércio eletrônico gostariam de utilizar esse tipo de sistema. Em nosso modelo assumimos essa sendo a porcentagem de clientes que utilizariam as EEAs.

O modelo tem dois tipos diferentes de clientes, que executam ações diferentes: o primeiro, é o cliente que escolhe pela entrega em domicílio, possui dois estados: um estado em que a entrega é realizada, ou seja, ele está em casa e recebe a mercadoria e um estado onde a entrega não é realizada, pois ele está ausente e não recebe a mercadoria. Adotamos uma probabilidade da entrega não acontecer na primeira tentativa de $25 \%$, na segunda tentativa de $60 \%$ e na terceira tentativa de 80\% (Arnold et al., 2018; Edwards et al., 2010). Segundo McLeod et al. (2006), as entregas que falharam na primeira vez teriam uma chance maior de falha na segunda ou terceira tentativa de entrega, dessa forma adotamos uma alta taxa de falhas para essas segundas e terceiras tentativas. 0 segundo tipo de cliente é o que escolhe receber sua entrega nas estações de entrega automática e possui 3 estados: Realizando a compra, Aguardando e Movimentando. Primeiro, ele faz o pedido e escolhe qual EEA ele quer receber a sua mercadoria. Nós assumimos que o Agente Cliente prefere receber a sua mercadoria na EEA mais próxima de sua casa, conforme sugerido em Iwan et al. (2016), Morganti et al. (2014) e Kedia et al. (2017). O software possui uma função chamada "neareast agent" (do inglês, agente mais próximo) que foi utilizada para identificar a EEA mais próxima daquele cliente. Após realizar essa escolha, o cliente fica aguardando a entrega do seu pedido, quando o pedido é entregue na EEA ele recebe então uma 
mensagem de "Pedido Entregue" enviada pelo Agente Caminhão. 0 cliente então se desloca até o EEA para retirar o seu pedido, e então retorna para casa.

As EEA no modelo interagem com a transportadora e com os clientes, que entregam e recebem os pedidos nestes locai, respectivamente. As EEA são um tipo de Agente inanimado que possuem apenas uma localização, e tem como objetivo hospedar o maior número de pedidos possíveis.

A quantidade de EEA representada no modelo seguiu a proporção de 5 para cada 100.000 habitantes, proporção utilizada nos locais onde estes sistemas já estão em funcionamento (Morganti et al., 2014; Weltevreden, 2008). Com relação a localização, segundo Weltevreden (2008) os supermercados estão entre os principais locais onde são instalados esses sistemas. Como essa também foi a preferência declarada pelos cliente do comércio eletrônico em Oliveira, et al. (2017) entre os supermercados existentes 5 geograficamente distantes para a operação dos EEA.

\section{Pedidos e modelagem da Loja}

Os pedidos são recebidos e gerenciados pelo Agente Loja. Cada pedido possui um determinado volume e peso e um endereço, que depende da escolha do cliente: pode ser sua casa ou a EEA. Assim que os pedidos vão sendo realizados, a loja inicia o processo de formação da carga, (a carga neste modelo contém uma coleção de pedidos). Este processo obedece dois fatores: a capacidade do caminhão e o tempo. 0 tempo refere-se a quantos dias um cliente espera para receber o seu pedido e foi utilizado o tempo médio informado pelo (Ebit, 2018). Assim que um desses fatores é atendido, uma carga é enviada para a transportadora. 0 Agente Loja ainda utiliza uma heurística para montar a carga, ou seja, os pedidos são separados em uma carga de acordo com a proximidade dos clientes e o Agente ainda separa na mesma carga os pedidos referentes a mesma EEA. Consequentemente, essa heurística melhora a rota realizada pelo caminhão. Os pedidos não entregues têm prioridade perante os outros pedidos e são alocados na carga do dia seguinte, pois obedecem a política das três tentativas.

\section{Transportadora}

A transportadora gerencia um número específico de caminhões para realizar a entrega da loja. Ela possui um centro de distribuição que está situado próximo à Avenida do Contorno. 0 processo de modelagem foi realizado a partir das ações do caminhão, que inicia a simulação no centro de distribuição aguardando a formação da carga. Se existe uma carga, o Agente Loja envia uma mensagem para Agente Caminhão "Iniciar Entrega".

A rota do caminhão segue o princípio do algoritmo do vizinho mais próximo, sua regra consiste em sair do ponto de partida $v_{i}$ e encontrar sempre o nó mais próximo $v_{k}$ que ainda não foi visitado. Continua-se assim até que todos os nós tenham sido visitados, e por fim retorna-se ao ponto de partida $v_{k}$ (Bodin, 1983). 0 ponto de partida neste modelo é o centro de distribuição (CD) da transportadora, ao sair do CD o Agente Caminhão faz uma lista de destinos, pois, como citado, os pedidos são entregues tanto na casa dos clientes como em pontos de EEA. A partir desta lista, o algoritmo identifica qual o destino que está mais próximo e se desloca até ele. Na situação em que o destino mais próximo é um ponto de EEA o Agente Caminhão verifica entre os outros pedidos da carga se existe outro com este mesmo destino e então descarrega e distribui todos os pedidos nos armários. Após esse processo ele envia uma mensagem de "Pedido Entregue" ao Agente Cliente para que ele possa retirar seu pedido. Já quando o destino é a casa 
do Agente Cliente, se o cliente estiver em casa o pedido é entregue; se o cliente estiver ausente o pedido retorna ao $\mathrm{CD}$ para uma posterior tentativa de entrega, até o número de tentativas ser igual a 3.

Os tempos de entrega incluem tempo de parada do caminhão e descarregamento. No caso do EEA, o tempo de descarregamento é função do número de pedidos entregues. Quando ocorre uma falha na entrega o único tempo contado é o de parada. Por fim, após percorrer a sua rota, o Agente Caminhão retorna ao $C D$, e aguarda até que outra carga seja enviada a ele. sua rota, o Agente Caminhão retorna ao CD, e aguarda até que outra carga seja enviada a ele.

\subsection{Modelo Computacional}

O modelo foi implementado no software Anylogic $®$, por apresentar a funcionalidade de MSBA e mapa com Sistema de Informação Geográfica (SIG- também conhecido como GIS acrónimo inglês de Geographic Information System). Essa funcionalidade permite que a simulação contenha os dados do ambiente georreferenciados, e ainda é capaz de definir e recuperar a localização atual no mapa de um agente, mover o agente com a velocidade especificada de um local para outro ao longo das rotas existentes (Anylogic, 2018). A Figura 1 apresenta o ambiente de simulação.

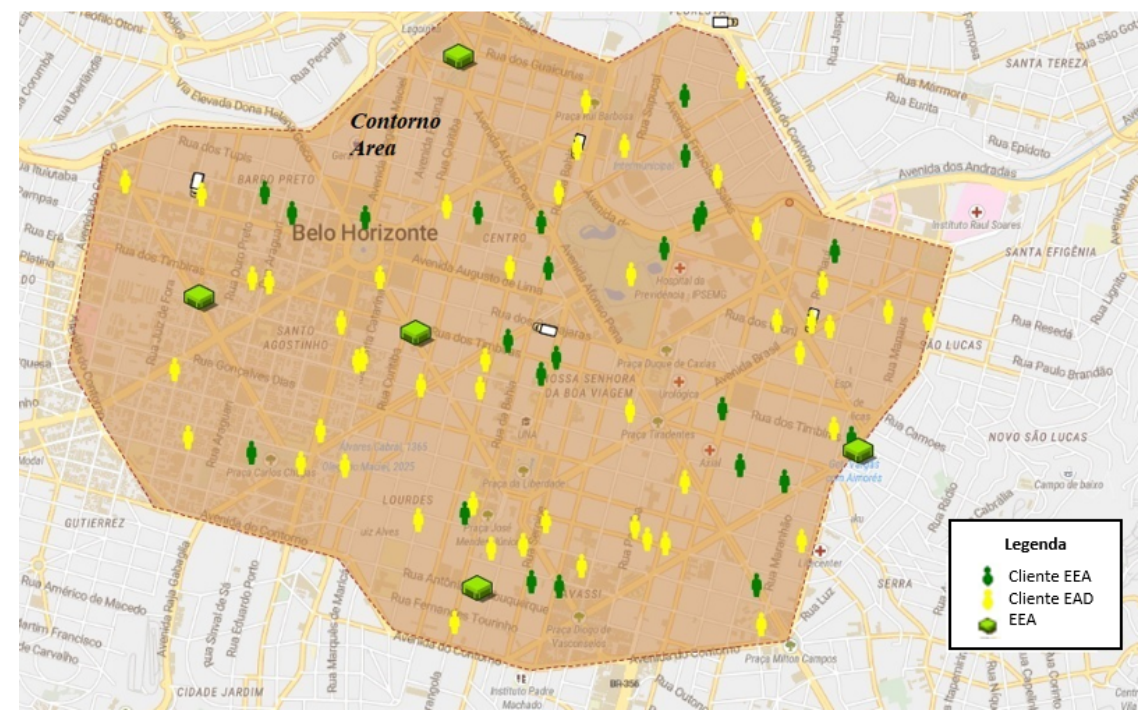

Figura 1. Ambiente de Simulação

Tabela 1 - Parâmetros de entrada

\begin{tabular}{lll}
\hline Agente & Parâmetro & Quantidade \\
\hline \multirow{4}{*}{ Caminhão } & Número de caminhões & 06 \\
& Peso & $1500 \mathrm{~kg}$ \\
& Volume & $10 \mathrm{~m}^{3}$ \\
& Velocidade & $12 \mathrm{~km} / \mathrm{h}$ \\
& Tempo de parade & 15 minutos \\
& Tempo de descaregamento & 8 minutos \\
\hline \multirow{3}{*}{ Clienter } & Localização & Aleátorio \\
& Escolha de entrega & Casa ou EEA \\
& Quantidade de pedidos & $1 /$ Ano \\
Pedidos & Média de tempo receber pedido & 9 dias \\
& Volume & triangular(0.1, 1, 0.6) $\mathrm{m}^{3}$ \\
\hline Fonte: Ebit (2018), (Oliveira, & Q 2014) e (Oliveira et al., 2017) & triangular(5, 20, 15) $\mathrm{kg}^{3}$
\end{tabular}


Os dados de entrada apresentados na Tabela 1 foram obtidos com as transportadoras locais e em pesquisa anterior de Oliveira et al. (2017), Oliveira (2014) e Ebit (2018). 0 parâmetro de entrada velocidade do caminhão, foi identificado junto as transportadoras entrevistadas que para entregas da última milha a velocidade média em regiões urbanas está em torno de 12 $\mathrm{km} /$ hora. Adotou-se este valor pois é o valor que tem sido utilizado por pesquisadores da área (Arnold et al., 2018; Masteguim et al., 2018). Os tempos de descarregamento e de parada, foram obtidos por meio de entrevistas com os motoristas da transportadora. Este valor foi confrontado com o indicado em estudo realizado por Oliveira (2014) na região estudada, e por se tratar de um valor próximo foi utilizado nas simulações.

Foram simulados 4 cenários variando a situação de reentrega e a implantação de EEAs:

Cenário 1 - Neste cenário, todos os pedidos devem ser entregues em casa e existe a política das três tentativas de entrega. Quando o cliente não está em casa para receber o pedido e este não é entregue, podem acontecer mais duas tentativas de entrega por dois dias consecutivos.

Cenário 2 - Agora há uma porcentagem de clientes que opta por receber suas entregas nas EEA. Permanecem as entregas EAD e, para este caso, as três tentativas de entrega.

Cenário 3 - Os clientes continuam a escolher onde preferem receber as suas entregas. Contudo, para as entregas à domicílio, se a entrega não for realizada na primeira tentativa, o entregador leva a encomenda com ele para o $\mathrm{CD}$, que avisará aos clientes para que eles se direcionem até o CD para recolher suas encomendas.

Cenário 4 - Neste caso, os clientes ainda podem escolher onde preferem receber as suas entregas. Contudo, se a entrega EAD não for realizada na primeira tentativa, o entregador deixa a encomenda no EEA mais próximo da casa do cliente e avisa ao cliente que o seu pedido está no EEA aguardando a retirada.

O cenário 1 é o mais próximo da situação atual de entregas, este cenário será utilizado para comparação com os demais cenários. Com o cenário 2 pretende-se avaliar qual o impacto se tem somente com a implantação ao implantar as EEAs. Nos cenários 3 e 4, simulou-se uma situação em que as três tentativas de entrega são excluídas. No cenário 3, os pedidos com falha devem ser buscados pelos clientes no centro de distribuição. 0 quarto cenário difere do cenário 3 no que se refere ao local de entrega de pedidos com falha, que se tornam EEAs. 0 principal objetivo aqui é analisar a distância percorrida pelos clientes para buscar seus pedidos com falha e verificar os resultados locais na menor distância.

\section{RESULTADOS DA SIMULAÇÃO}

Para cada cenário, foram simulados um ano de entregas, com 15 replicações cada. A Tabela 2 apresenta a quantidade de pedidos entregues e o número de reentregas em cada cenário.

Tabela 2 - Quantidade de pedidos entregues e reentregas

\begin{tabular}{lllll}
\hline & \multicolumn{2}{l}{ Com três tentativas } & \multicolumn{2}{l}{ Sem três tentativas } \\
\hline & Cenário 1 & Cenário 2 & Cenário 3 & Cenário 4 \\
Total de pedidos entregues em casa & 25053 & 14300 & 12170 & 12216 \\
Total de pedidos falhos & 3408 & 1943 & 4057 & 4068 \\
Total de pedidos EEA & 0 & 12215 & 12235 & 16293 \\
Número de reentregas & 14726 & 8401 & 4057 & 4068 \\
Total de pedidos entregues & 25053 & 26515 & 28462 & 28464 \\
\hline
\end{tabular}

Ao se comparar o cenário 1 com o 2 (Tabela 2), que simula a implantação da EEA e mantém as três tentativas de entrega, é possível aumentar em $6 \%$ o número de pedidos entregues. 
Os cenários que simulam a política das três tentativas apresentam um número de reentregas alto. Contudo, o cenário 2 que simula a implantação das EEA conseguiu reduzir em $43 \%$ o número de reentregas, quando comparado com o cenário 1.

Os cenários 3 e 4 não consideram a política das três tentativas, comparando-os com o cenário 1 , apresentam uma redução de $72 \%$ do número de tentativas de entregas. Isso acontece porque nestes cenários somente na primeira tentativa de entrega pode acontecer a falha. Os pedidos falhos do cenário 3 e 4, são os pedidos que o entregador não conseguiu entregar em casa. Entretanto, nestes cenários, após a primeira tentativa falha, os clientes conseguem retirar os seus pedidos ou no CD (cenário 3) ou no EEA mais próxima (cenário 4). Nestes cenários todos os pedidos são entregues. A Tabela 3 apresenta a distância percorrida por cada um dos 6 caminhões nos quatro cenários.

Tabela 3 - Distâncias percorridas pelos caminhões

\begin{tabular}{lllll}
\hline & \multicolumn{2}{l}{ Com Três tentativas } & \multicolumn{2}{l}{ Sem três tentativas } \\
\hline Distâncias (km) & Cenário 1 & Cenário 2 & Cenário 3 & Cenário 4 \\
Caminhão 1 & 4966 & 4520 & 2968 & 3745 \\
Caminhão 2 & 4577 & 3974 & 2833 & 3671 \\
Caminhão 3 & 4189 & 3714 & 2587 & 3192 \\
Caminhão 4 & 3931 & 3599 & 2320 & 2601 \\
Caminhão 5 & 3814 & 3304 & 2179 & 2367 \\
Caminhão 6 & 3847 & 2920 & 1984 & 2151 \\
TOTAL & $\mathbf{2 5 3 2 4}$ & $\mathbf{2 2 0 3 1}$ & $\mathbf{1 4 8 7 2}$ & $\mathbf{1 7 7 2 8}$ \\
\hline
\end{tabular}

Os cenários 2, 3 e 4 apresentam uma redução dos quilômetros percorridos pelos caminhões em $13 \%, 41 \%$ e $29 \%$, respectivamente, quando comparados com o cenário 1 . Esse efeito era esperado devido à redução no número de locais de entrega e tentativas de devolução (Forkert e Eichhorn, 2007). Esse resultado corrobora o encontrado por Cardenas et al., (2016) e Esser e Kurte, (2007), que obtiveram uma redução no quilômetros viajados por veículo (vehicle-kilometers traveled-VKT), com um número pequeno de pontos de coleta. Analisando isoladamente, a implantação da EEA seria suficiente para reduzir o a distância percorrida. Quando excluímos a política das três tentativas, obtemos resultados ainda melhores. As distâncias maiores no cenário 4, em comparação com o cenário 3, se explica pelo fato de que no cenário 4, quando não ocorre a entrega, o entregador precisa se dirigir ao EEA mais próximo para deixar a encomenda, sendo que esse EEA pode não estar na sua rota ou até já ter sido visitado anteriormente. No cenário 3, quando o pedido não é entregue, ele é levado junto com os demais pedidos não entregues de uma única vez ao $\mathrm{CD}$, que usualmente é o ponto de destino da rota e não acarreta em nenhuma parada adicional.

Analisamos também o número de quilômetros percorridos pelos clientes ao buscar suas mercadorias (Tabela 4). Os Clientes EAD são aqueles que inicialmente optam por receber seus pedidos em casa; os Clientes EEA são aqueles que escolhem receber seus pedidos nas estações de entrega.

A distância média percorrida pelos clientes EEA estão entre $1.2 \mathrm{~km}$ e $1.6 \mathrm{~km}$. De modo contrário, temos maiores distâncias médias percorridas pelos Clientes EAD no cenário 3, que sugerem o uso de modos motorizados para recolher seus pedidos. No pior caso, resultaria em 338 viagens por mês ao CD para retirar os pedidos falhos. Quando o local onde os clientes buscam suas entregas falhas passa a ser o EEA (cenário 4), temos uma redução de $46 \%$ da distância 
individual média percorrida. Este é um ganho muito positivo, pois, quanto menor a distância maior mais propensos estarão os clientes a buscarem suas encomendas por meios não motorizados é o que indica o estudo de Arnold et al. (2018) e Cardenas et al. (2016).

Tabela 4 - Distância percorrida por clientes ao buscar suas mercadorias no CD e EEA

\begin{tabular}{cllllll}
\hline & \multicolumn{3}{c}{ Clientes EAD } & \multicolumn{3}{c}{ Clientes EEA } \\
\hline Distâncias(km) & Cenário 2 & Cenário 3 & Cenário 4 & Cenário 2 & Cenário 3 & Cenário 4 \\
\hline CD & 11868 & & & & \\
\hline EEA & & 5625 & 19677 & 19890 & 19812 \\
\hline Média & 3 & 1.4 & 1.6 & 1.6 & 1.2 \\
\hline
\end{tabular}

Embora a distância percorrida seja a principal variável analisada nos trabalhos sobre a implantação dos pontos de coletas (Cardenas et al., 2016; Esser e Kurte, 2007; McLeod et al., 2006), observa-se que ao consolidar as cargas e excluir as tentativas de entrega o número de cargas entregues por caminhão teve uma redução considerável.

No cenário 2, onde se tem a implantação do EEA, houve uma redução de 12\% do número de cargas transportadas por ano quando comparado com o cenário 1. Nos cenários onde excluímos a terceira tentativa, temos uma redução $28 \%$ no número de cargas. Dessa forma, a fim de obter uma utilização maior dos caminhões, simulamos a alteração na quantidade de caminhões, mantendo inalterados os outros parâmetros de entrada. A melhor configuração obtida, em termos de redução do número de caminhões, foi a de cinco caminhões para o cenário 2 e quatro caminhões para os cenários 3 e 4, obedecendo as janelas de tempo da transportadora. Assim os cenários já apresentados foram novamente simulados, alterando o número de caminhões para cinco (cenário 2) e quatro (cenários 3 e 4).

A nova simulação não altera o número de cargas transportada por ano, mas aumenta a utilização de cada caminhão. Não houve alteração significativa na distância percorrida pelos caminhões nestas novas simulações, quando comparadas com as simulações anteriores (seis caminhões). Os demais resultados percentuais discutidos anteriormente com relação a distância percorrida pelos clientes, números de pedidos entregues e falhos permanecem similares.

Conforme indicado pela MSBA, cada agente possui um comportamento e um ou mais objetivos a atingir. Contudo, esses objetivos trazem ganhos maiores ou menores, dependendo das regras a serem observadas em cada cenário. Como o cenário 1 é o cenário mais próximo da situação real, os ganhos para cada agente foram calculados em comparação com este cenário (Tabela 5).

Tabela 5 - Resumo dos ganhos obtidos por cada agente na simulação

\begin{tabular}{llll}
\hline & \multicolumn{1}{c}{ Cenário 2 } & \multicolumn{1}{c}{ Cenário 3 } & \multicolumn{1}{c}{ Cenário 4 } \\
\hline \multirow{2}{*}{ Transportadores } & Redução de 13\% VKT & Redução 41\% VKT \\
& Redução de 1 Caminhão & Redução de 2 Caminhões & Redução de 29 \% VKT \\
& Redução de 2 Caminhões \\
\hline \multirow{2}{*}{ Loja E-commerce } & $\begin{array}{l}\text { Aumentou número de } \\
\text { pedidos entregues em 5.8\% }\end{array}$ & $\begin{array}{l}\text { Aumentou número de } \\
\text { pedidos entregues em 13.5\% }\end{array}$ & $\begin{array}{l}\text { Aumentou número de pedidos en- } \\
\text { tregues em 13.5\% }\end{array}$ \\
\hline EEAs & Hospedou 12215 pedidos & Hospedou 12235 pedidos & Hospedou 16293 pedidos \\
\hline \multirow{4}{*}{ Clientes } & $93 \%$ Pedidos entregues & $100 \%$ Pedidos entregues & $100 \%$ Pedidos entregues \\
& Número médio de km & Número médio de km percorridos & Número médio de km percorridos \\
& percorridos Clientes & Clientes EAD 3 km & Clientes EAD 1.4 km \\
& EEA 1.6 km & Número médio de km percorridos & Número médio de km percorridos \\
& & Clientes EEA 1.6 km & Clientes EEA 1.2 km \\
\hline
\end{tabular}


O cenário 1 apresenta a maior distância percorrida, o maior número de pedidos em reentrega e quando comparado com o cenário 2 o maior número de pedidos não entregues, uma vez que os pedidos com falha na primeira tentativa no cenário 3 e 4, são encaminhados ao CD ou as EEAs para a posterior retirada do cliente. Um pedido não entregue pode prejudicar a reputação da loja do e-commerce e ainda demandar um novo re-envio que onerará a loja ou o cliente.

Visto isto os cenários que apresentam os melhores resultados são os cenários 3 e 4. Embora o cenário 3 apresente o menor número de quilômetros percorridos pelos caminhões, o cenário 4 apresenta distâncias menores percorridas pelos clientes ao buscar seus pedidos nos EEA e ganhos maiores para os supermercados que os abrigam. Dessa forma o cenário 4 onde há a maior utilização dos EEA, apresenta melhores resultados globais.

\section{DISCUSSÕES}

O cenário 1 é o que mais se assemelha à situação atual, onde as entregas são todas AD e nas quais podem ocorrer até três tentativas de entrega, foi o que apresentou os piores resultados, demanda de mais caminhões, maior distância percorrida, maior número de entregas não realizadas e consequente maior número de reentregas. Em pesquisa realizada com 300 varejistas do e-commerce do Reino Unido, EUA, e Alemanha o custo de uma entrega não realizada foi estimado por eles em US\$17,78 (PCAPredict, 2018). No entanto, os varejistas apontam que este valor é subestimado, já que não leva em conta o dano que uma entrega não realizada pode causar à reputação da marca a possível perda de clientes existentes e a dificuldade de adquirir novos clientes. Dessa forma, as EEA, além de reduzir o custo das reentregas, podem contribuir para melhorar a reputação das lojas do e-commerce.

Além de contribuir para a redução do número de reentregas a implantação dos EEA pode gerar uma renda extra para os s supermercados que as hospedam. Segundo Weltevreden (2008), para cada pedido entregue em um ponto de coleta o varejista recebe de US $\$ 0.28$ a US\$0.49, dessa forma os supermercados ganhariam na ordem de U\$1,600 cada. Esse é um valor pequeno, pois é calculado estimando a entrega de 15 pacotes/dia. Entretanto, os armários têm capacidade para acomodar de 40 a 100 pacotes/dia, o que pode aumentar em quase 7 vezes esse valor, dependendo do crescimento da demanda. Ainda, o fluxo extra de pessoas que visitam a loja para utilizar as EEA, pode aumentar o número de vendas dos supermercados. Segundo Iwan et al. (2016), a cada quatro usuários dos EEA, um realiza compras ao retirar seus pedidos. De acordo com Oliveira et al. (2017) os clientes do comércio eletrônico da cidade de Belo horizonte demonstraram disposição de pagar $\mathrm{R} \$ 0.50$ a mais por entrega para utilizar os EEA, valor que poderia contribuir para pagar os supermercados.

O cenário 4 apresenta uma alternativa melhor tanto para os clientes que escolheram receber suas encomendas nas EEAs quanto para aqueles que utilizariam o sistema para buscar uma entrega não realizada. Segundo pesquisa realizada por McLeod et al. (2006), a maior parte dos clientes preferem retirar sua entrega falha em um EEA ao invés de ir busca-las no $\mathrm{CD}$, quando isto representar uma economia em tempo e distância percorrida. Esta distância menor percorrida pelos clientes no cenário 4 , contribui para equilibrar a distância percorrida a mais pelos caminhões neste cenário em comparação com o cenário 3.

Reduzir o número de caminhões é um benefício potencial das estações de entrega automática que é ainda pouco explorado na literatura, pois a maior parte dos estudos analisa os resultados em termos de distância percorrida pelos caminhões e clientes (Cardenas et al., 2017; Esser e 
Kurte, 2007; Kedia et al., 2017; McLeod et al., 2006; Weltevreden, 2008). A redução do número de caminhões resultará ainda em um número menor de motoristas, menor gasto com manutenção/impostos dos veículos e menor custo da transportadora como um todo.

Esses resultados destacam que a auto-coleta do cliente traz ganhos para mais de um stakeholder. No entanto, a promoção da auto-coleta pode ser difícil, já que a maioria dos clientes está acostumada à comodidade das entregas em casa. Uma forma de promover o maior uso das EEAs seria oferecendo de reduções no valor do frete cobrado.

Um dos efeitos negativos do uso das EEAs é a geração de viagens exclusivas de clientes buscando seus pedidos de carro (Morganti, Seidel, et al. 2014). No entanto, de acordo com Arnold et al. (2018) e Cardenas et al. (2016) se a EEA estiver a uma distância pequena, menos pessoas usarão seus carros para buscar as encomendas. Ao mesmo tempo, os clientes se sentirão mais dispostos a aceitar a opção de auto-coleta. Neste sentido Masteguim et al. (2018) atestam que a distância entre clientes e EEAs pode ser reduzida com a abertura de mais EEAs na cidade, o que requer um investimento significativo e que pode desestimular a implantação desta iniciativa, 0 envolvimento e patrocínio das autoridades públicas poderiam favorecer este processo.

No Brasil não se observa ainda os ganhos com consolidação, devido a pequena e recente utilização das EEAs, que estão ainda em teste na cidade de São Paulo, com poucos pontos. No entanto, segundo o responsável pelas operações, alguns ganhos já podem ser observados. Clientes que residem em zonas periféricas, por exemplo, onde as transportadoras não realizam o serviço de entrega, agora podem utilizar estes locais para receber suas mercadorias, aumentando assim a capilaridade das entregas e as vendas online.

\section{CONCLUSÕES}

Demonstramos neste estudo que a Modelagem e Simulação Baseada em Agentes é uma ferramenta útil para modelar o transporte urbano de mercadorias. No contexto das entregas do comércio eletrônico foi capaz de simular corretamente as interações entre os agentes e as ações tomadas por eles diante de diferentes situações. 0 cenário que mais se assemelha com a situação atual, onde as entregas são todas assistidas á domicilio e em que poderão ocorrer até três tentativas de entregas foi o que apresentou piores resultados. A implantação das estações de entrega automática traz melhorias para este cenário, em termos de redução das reentregas e distâncias percorridas pelos caminhões. Contudo, quando é excluída a possibilidade das três tentativas de entrega, os benefícios são ainda maiores.

Um efeito indesejado da implantação das estações de entrega automática é o aumento do número de viagens particulares dos clientes ao buscarem suas mercadorias. O MSBA mostrou que, ao buscar suas entregas falhas no centro de distribuição (Cenário 3), os clientes precisam percorrer longas distâncias, o que poderia aumentar o tráfego de veículos na região. Quando as EEAs são utilizadas para receber as entregas falhas, as distâncias percorridas pelos clientes podem ser reduzidas em até $46 \%$ podendo, em muitos casos, ser percorridas por bicicleta ou a pé. 0 mesmo ocorre com os clientes que escolhem inicialmente as EEAs para receber seus pedidos.

O MSBA mostrou ainda que as EEAs apresentam ganhos para as transportadoras que vão além da redução dos quilômetros percorridos. Quando simulamos cenários com as EEAs observamos reduções no número de caminhões entre 17\% a 33\%, dependendo da exclusão ou não da política das três tentativas. Isoladamente, tanto a implantação das EEAs quanto a exclusão das três tentativas não parecem benéficas para todas as partes interessadas. No entanto, a combinação de ambos os conceitos representa um sistema de entregas do comércio eltronico-B2C 
atraente para todos os envolvidos. A eficiência de tal sistema de entrega pode ser ainda mais aprimorada se mais clientes utilizarem as EEAs para receber suas encomendas.

Esses resultados destacam a importância de tratar as entregas do comércio eltronico-B2C por uma perspectiva global. Os diversos stakeholders seguem diferentes metas e estratégias. As entregas à domicilio são as preferidas pelos clientes e as EEAs são as que trazem maiores redução de custos para as transportadoras e lojas do e-commerce. Esse cenário de interesses contraditórios poderia ser melhorado com políticas de incentivo para que o cliente utilizasse a EEAs através, por exemplo, de um desconto na entrega ou outro tipo de bônus, monetário ou não.

Além disso, um diálogo objetivo entre as partes interessadas requer uma avaliação realista de possíveis cenários "what-if". Nós demonstramos que tal avaliação é possível ao se utilizar a simulação baseada em agentes. Por meio da simulação, foi possível modelar e avaliar diferentes estratégias de entrega, o que nos permitiu obter resultados comparáveis para cada agente. Pela comparação de diferentes cenários, constatamos que a implantação das EEAs e a exclusão das três tentativas, podem aumentar o número de pedidos entregues em 14\% e diminuir em 29\% a distância percorrida pelos caminhões.

A obtenção de dados é de crucial importância em estudos de simulação. Neste estudo, por se tratar de um modelo complexo que envolve vários stakeholders identificamos uma grande dificuldade para coletar informações. Estudos futuros que explorem essa questão, em especial com relação aos tempos de parada, descarregamento e velocidade trariam uma boa contribuição para a comunidade cientifica interessada nas entregas da última milha.

Finalmente, como visto em nossos resultados as EEAs representam uma alternativa promissora para reduzir os problemas das entregas à domicilio, pois quando estrategicamente localizadas, facilitam o acesso não motorizado. Contudo, ao se implementar uma rede de EEAs para o contexto brasileiro, questões de segurança devem aparecer como elemento crucial a ser considerado. 0 sucesso dessa solução depende: das lojas do $e$-commerce oferecerem e promoverem em seus websites essa opção para seus clientes; da aceitação dos clientes do comércio eletrônico, e do interesse dos varejistas em hospedarem esta solução. Incentivos a utilização das EEAs poderiam fazer parte de políticas públicas de mobilidade urbana, já que a sua utilização reduziria os efeitos negativos do transporte urbano de mercadorias na mobilidade.

\section{AGRADECIMENTOS}

Os autores agradecem a CAPES, ao CNPq e a FAPEMIG, pelo apoio financeiro concedido a diversos projetos que subsidiaram o desenvolvimento desse trabalho.

\section{REFERENCES}

Alho, A., Bhavathrathan, B. K., Stinson, M., Gopalakrishnan, R., Le, D. T., e Ben-Akiva, M. (2017) A multi-scale agent-based modelling framework for urban freight distribution. Transportation Research Procedia, 27, 188-196. doi:10.1016/j.trpro.2017.12.138

Alves, R., Lima, R. da S., Silva, K., Gomes, W., e González-Calderón, C. (2019) Functional and environmental impact analysis of urban deliveries in a Brazilian historical city. Case Studies on Transport Policy, 7(2), 443-452. doi:10.1016/j.cstp.2019.01.009

Anand, N., Van Duin, J. H. R., e Tavasszy, L. (2016) Framework for Modelling Multi-stakeholder City Logistics Domain Using the Agent based Modelling Approach. Transportation Research Procedia, 16(March), 4-15. doi:10.1016/j.trpro.2016.11.002

Anylogic. (2018) No Title. Obtido 28 de maio de 2018, de https://www.anylogicbrasil.com.br/

Arnold, F., Cardenas, I., Sörensen, K., e Dewulf, W. (2018) Simulation of B2C e-commerce distribution in Antwerp using cargo bikes and delivery points. European Transport Research Review, 10(1). doi:10.1007/s12544-017-0272-6

Bodin, L. (1983) Routing and Acheduling of vehicles and crews, the state of the art. Computers operational research, 10(2), 63-211.

Cardenas, I., Dewulf, W., Vanelslander, T., Smet, C., e Beckers, J. (2017) The e-commerce parcel delivery market and the implications of home B2C deliveries vs pick-up points. International Journal of Transport Economics, 44(2), 235-256. doi:10.19272/201706702004 
Cardenas, Ivan, Beckers, J., Vanelslander, T., e Verhetsel, A. (2016a) Spatial characteristics of failed and successful E- commerce deliveries in Belgian cities. ILS 2016, 6th International Conference on Information Systems, Logistics and Supply Chain, Bordeaux, France, 1-4 June, 1-10.

Cullinane, S. (2009) From bricks to clicks: The impact of online retailing on transport and the environment. Transport Reviews, 29(6), 759-776. doi:10.1080/01441640902796364

Davidsson, P., Henesey, L., Ramstedt, L., Törnquist, J., e Wernstedt, F. (2005) An analysis of agent-based approaches to transport logistics. Transportation Research Part C: Emerging Technologies, 13(4), 255-271. doi:10.1016/j.trc.2005.07.002

Dell'Amico, M., e Hadjidimitriou, S. (2012) Innovative Logistics Model and Containers Solution for Efficient Last Mile Delivery. Procedia - Social and Behavioral Sciences, 48, 1505-1514. doi:10.1016/j.sbspro.2012.06.1126

Ducret, R. (2014) Parcel deliveries and urban logistics : Changes and challenges in the courier express and parcel sector in Europe - The French case. Research in Transportation Business \&Management Final, 11, 15-22. doi:10.1016/j.rtbm.2014.06.009

Ebit. (2018) WEBSHOPPERS 37a Edição 2018., 45. Obtido de http://www.fecomercio.com.br/public/upload/editor/pdfs/ws37_imprensa.pdf

Ebit. (2019) 39a Edição 2019. WEBSHOPPERS 39a EDIÇÃO.

Edwards, J., Mckinnon, A., Cherrett, T., Mcleod, F., e Song, L. (2010) Carbon Dioxide Benefits of Using Collection - Delivery Points for Failed Home Deliveries in the United Kingdom., 136-143. doi:10.3141/2191-17

Elbert, R., e Friedrich, C. (2018) SIMULATION-BASED EVALUATION OF URBAN CONSOLIDATION CENTERS CONSIDERING URBAN ACCESS REGULATIONS. Proceedings of the 2018 Winter Simulation Conference, 2827-2838. doi:10.1017/CB09781107415324.004

EMARKETER. (2019) Global Ecommerce 2019. Obtido 20 de agosto de 2019, de https://www.emarketer.com/content/globalecommerce-2019

Esser, K., e Kurte, J. (2007) Strategies for Optimizing Pick-up and Delivery Traffic of Internet Commerce - Packstations in Cologne (OPTIMAL). KE-Consult: Cologne, Germany.

Firdausiyah, N., Taniguchi, E., e Qureshi, A. G. (2019) Modeling city logistics using adaptive dynamic programming based multi-agent simulation. Transportation Research Part E: Logistics and Transportation Review, 125(March), 74-96. doi:10.1016/j.tre.2019.02.011

Forkert, S., e Eichhorn, C. (2007) Alternative Solution for Home Delivery. Niches Transport.

Fox, M. S., Barbuceanu, M., e Teigen, R. (2000) Agent-oriented supply-chain management. International Journal of Flexible Manufacturing Systems, 12(2), 165-188. Obtido de http://www.scopus.com/inward/record.url?eid=2-s2.00034171704\&partnerID=40\&md5=f8843fedf34ca651bf517769b650beff

Holguín-Veras, J., Sánchez-Díaz, I., e Browne, M. (2016) Sustainable Urban Freight Systems and Freight Demand Management. Transportation Research Procedia, 12(June 2015), 40-52. doi:10.1016/j.trpro.2016.02.024

IBGE. (2010) Censo Demográfico. Obtido de http://censo2010.ibge.gov.br

Iwan, S., Kijewska, K., e Lemke, J. (2016) Analysis of parcel lockers ' efficiency as the last mile delivery solution - the results of the research in Poland. Transp. Res. Procedia, 12(June 2015), 644-655. doi:10.1016/j.trpro.2016.02.018

Kedia, A., Kusumastuti, D., e Nicholson, A. (2017) Acceptability of collection and delivery points from consumers' perspective: A qualitative case study of Christchurch city. Case Studies on Transport Policy, 5(4), 587-595. doi:10.1016/j.cstp.2017.10.009

Liu, C., Wang, Q., e Susilo, Y. O. (2017) Assessing the impacts of collection-delivery points to individual's activity-travel patterns: A greener last mile alternative? Transportation Research Part E: Logistics and Transportation Review. doi:10.1016/j.tre.2017.08.007

Maere, B. De. (2017) Ecological and economic impact of automated parcel lockers vs home delivery RESEARCH PAPER 20162017., (June).

Marcucci, E., Le Pira, M., Gatta, V., Inturri, G., Ignaccolo, M., e Pluchino, A. (2017) Simulating participatory urban freight transport policy-making: Accounting for heterogeneous stakeholders' preferences and interaction effects. Transportation Research Part E: Logistics and Transportation Review, 103, 69-86. doi:10.1016/j.tre.2017.04.006

Masteguim, R. de F., Cunha, C. B. da, e Ponce, E. (2018) Modelagem e análise dos impactos econômicos na utilização de pontos de coleta na distribuição urbana de e-commerce: o caso de são paulo. $32^{\circ}$ Congresso de Pesquisa Ensino e Transporte da ANPET, 2236-2248.

McLeod, F., Cherrett, T., e Song, L. (2006) Transport impacts of local collection/delivery points. International Journal of Logistics Research and Applications, 9(3), 307-317. doi:10.1080/13675560600859565

Morganti, E., Seidel, S., Blanquart, C., Dablanc, L., e Lenz, B. (2014) The impact of e-commerce on final deliveries : alternative parcel delivery services in France and Germany. Transportation Research Procedia, 4(0), 178-190. doi:10.1016/j.trpro.2014.11.014

Oliveira, L. K. De. (2014) Diagnóstico das vagas de carga e descarga para a distribuição urbana de mercadorias: um estudo de caso em Belo Horizonte. Journal of Transport Literature. doi:10.1590/S2238-10312014000100009

Oliveira, L. K. de, Lessa, D. A., Oliveira, E., e Gregório Calazans, B. F. (2017) Multi-agent modelling approach for evaluating the city logistics dynamic in a vulnerability situation: An exploratory study in Belo Horizonte (Brazil). Transportation Research Procedia, 25, 1046-1060. doi:10.1016/j.trpro.2017.05.478 
Oliveira, L. K. de, Morganti, E., Dablanc, L., e Oliveira, R. L. M. de. (2017) Analysis of the potential demand of automated delivery stations for e-commerce deliveries in Belo Horizonte, Brazil. Research in Transportation Economics, 65, 34-43. doi:https://doi.org/10.1016/j.retrec.2017.09.003

Oliveira, J. B., Lima, R. S., e Montevechi, J. A. B. (2016) Perspectives and relationships in Supply Chain Simulation: A systematic literature review. Simulation Modelling Practice and Theory, 62(March 2018), 166-191. doi:10.1016/j.simpat.2016.02.001

PCAPredict. (2018) Fixing Failed Deliveries: Improving Data Quality in Retail. Worcester, UK.

Roorda, M. J., Cavalcante, R., McCabe, S., e Kwan, H. (2010) A conceptual framework for agent-based modelling of logistics services. Transportation Research Part E: Logistics and Transportation Review, 46(1), 18-31. doi:10.1016/j.tre.2009.06.002

Schroeder, S., Zilske, M., Liedtke, G., e Nagel, K. (2012) Towards a Multi-Agent Logistics and Commercial Transport Model: The Transport Service Provider's View. Procedia - Social and Behavioral Sciences, 39, 649-663. doi:10.1016/j.sbspro.2012.03.137

Song, L., Cherrett, T., e Guan, W. (2011) Implications of collection/delivery points for transport and logistics. OR Insight, 24(4), 231. doi:10.1057/ori.2011.9

Song, L., Cherrett, T., McLeod, F., e Guan, W. (2009) Addressing the Last Mile Problem. Transportation Research Record: Journal of the Transportation Research Board, 2097(2097), 9-18. doi:10.3141/2097-02

Sopha, B. M., Siagian, A., e Asih, A. M. S. (2016) Simulating Dynamic Vehicle Routing Problem Using Agent-Based Modeling and Simulation. 2016 Ieee International Conference on Industrial Engineering and Engineering Management (Ieem). Bali, Indonesia, 4-7 December, 1335-1339. doi:10.1109/IEEM.2016.7798095

Taniguchi, E., Thompson, R. G., e Yamada, T. (2012) Emerging Techniques for Enhancing the Practical Application of City Logistics Models. Procedia - Social and Behavioral Sciences. doi:10.1016/j.sbspro.2012.03.087

Teo, J., Taniguchi, E., e Qureshi, A. (2012) Evaluation of Distance-Based and Cordon-Based Urban Freight Road Pricing in ECommerce Environment with Multiagent Model. Transportation Research Record: Journal of the Transportation Research Board, 2269(2269), 127-134. doi:10.3141/2269-15

van Heeswijk, W., Larsen, R., e Larsen, A. (2019) An urban consolidation center in the city of Copenhagen: A simulation study. International Journal of Sustainable Transportation, 13(9), 675-691. doi:10.1080/15568318.2018.1503380

Van Kolck, A. H. (2010) Multi-agent model for an Urban Distribution Centre. Delft University of Technology, Delft, Netherlands.

Visser, J., Nemoto, T., e Browne, M. (2014) Home Delivery and the Impacts on Urban Freight Transport: A Review. Procedia Social and Behavioral Sciences, 125, 15-27. doi:10.1016/j.sbspro.2014.01.1452

Wang, X., Zhan, L., Ruan, J., e Zhang, J. (2014) How to choose "last mile" delivery modes for E-fulfillment. Mathematical Problems in Engineering, 2014(1). doi:doi:10.1155/2014/417129

Wangapisit, O., Taniguchi, E., e Qureshi, A. G. (2013) Multi-Agent Modelling Systems for Evaluating Urban Freight Policy Measures on Parking Space Restriction. General Proceedings of the 13th World Conference on Transport Research Society (15-18 July 2013, Rio de Janeiro, Brazil), 1-12.

Weltevreden, J. W. J. (2008) B2c e-commerce logistics: the rise of collection-and-delivery points in The Netherlands. International Journal of Retail \& Distribution Management, 36(8), 638-660. doi:10.1108/09590550810883487

Weltevreden, J. W. J., e Rotem-Mindali, 0. (2009) Mobility effects of b2c and c2c e-commerce in the Netherlands: a quantitative assessment. Journal of Transport Geography, 17(2), 83-92. doi:10.1016/j.jtrangeo.2008.11.005 